\title{
Thymectomy using the subxiphoid approach
} \author{
Japan
}

Motoki Yano, MD, Satoru Moriyama, MD, Hiroshi Haneda, MD, and Ryoichi Nakanishi, MD, Nagoya,
Video clip is available online.

A 66-year-old woman was referred to our hospital due to the finding of an anterior mediastinal mass on chest computed tomography $(\mathrm{CT})$. The mass, with a maximal tumor diameter measuring $2.6 \mathrm{~cm}$, was enhanced by contrast $\mathrm{CT}$ and diagnosed to be thymoma, although the patient was negative for anti-acetylcholine receptor antibody. Reconstructed 3-dimensional images revealed a sufficient distance $(3.0 \mathrm{~cm})$ between the mass and the left brachiocephalic vein (Figure 1). Therefore, thymectomy using the subxiphoid approach was initiated under general anesthesia with the patient in the lithotomy position using a single-lumen tracheal tube. A 3-cm transverse skin incision was made 1 to $2 \mathrm{~cm}$ below the lower edge of the xiphoid. The anterior layer of the rectus sheath was cut and dissected on the reverse side of the xiphoid by the finger. A GelPOINT Mini (Applied Medical, Rancho Santa Margarita, Calif) (access platform of single-port surgery) was inserted and fixed. A long 5-mm thoracoscope with a 30-degree angle was inserted through a port of the GelPOINT Mini. Using carbon dioxide insufflation of $8 \mathrm{~mm}$ $\mathrm{Hg}$, the view was rapidly extended. A LigaSure Maryland (Covidien, Mansfield, Mass) was also inserted through the second port of the GelPOINT Mini. The connective tissue between the sternum and pericardium was cut by the LigaSure Maryland (Video 1). The bilateral pleura and the lower edges of the thymus were revealed by repeating this procedure. The pleura was opened by the tip of the LigaSure Maryland. A thoracic 5-mm port was inserted at the fifth intercostal space on the right anterior axillary line. The lower edge of the thymus, the connecting pericardial fat, and the right phrenic nerve were easily visualized through the thoracic port. A portion of the

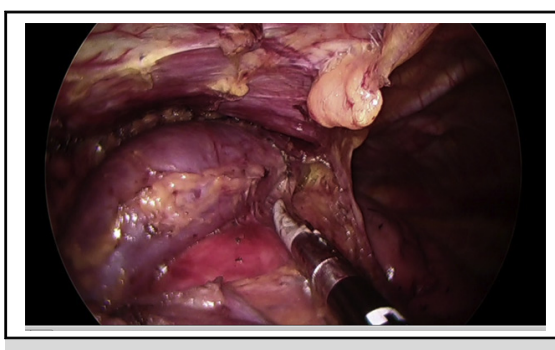

An intraoperative view of thymectomy.

Central Message

Thymectomy is regarded as a standard operation for thymoma. We have recently established a minimally invasive thymectomy technique using the subxiphoid approach and videoassisted thoracic surgery.

See Editorial Commentary page 280.

pericardial fat tissue surrounding the lower edge of the thymus is typically resected. Dissection of the lateral edge of the thymus was performed using the LigaSure Maryland running along the phrenic nerve. The exposure of the superior vena cava and the internal thoracic vein facilitated the exposure of the left brachiocephalic vein. The thymic artery was easily exposed as a branch from the internal thoracic artery and cut by the LigaSure Maryland. A SILS Clinch (Covidien) was inserted through the second port of the GelPOINT Mini. This Clinch was used for traction of the thymus. Although the thoracoscope and the Clinch were mainly inserted through the thoracic port and the second port of the GelPOINT Mini, respectively, their positions were changed as necessary. The bilateral upper edges of the thymus were dissected by LigaSure Maryland and the junction of the inferior thyroid vein and left brachiocephalic vein was recognized. Further traction of the thymus to the left and caudal direction exposed the thymic veins. The thymic veins were carefully exposed, sealed, and cut by the LigaSure Maryland. The left thymic artery was cut following dissection of the left side of the thymus. Finally, thymectomy was completed (Figure 2). A thoracic drain was inserted and set to the anterior mediastinum through the right thoracic port. The blood loss was minimal and the operation time was 96 minutes. The chest drain was removed on the first postoperative day. The patient was discharged on the third postoperative day without any postoperative complications. 


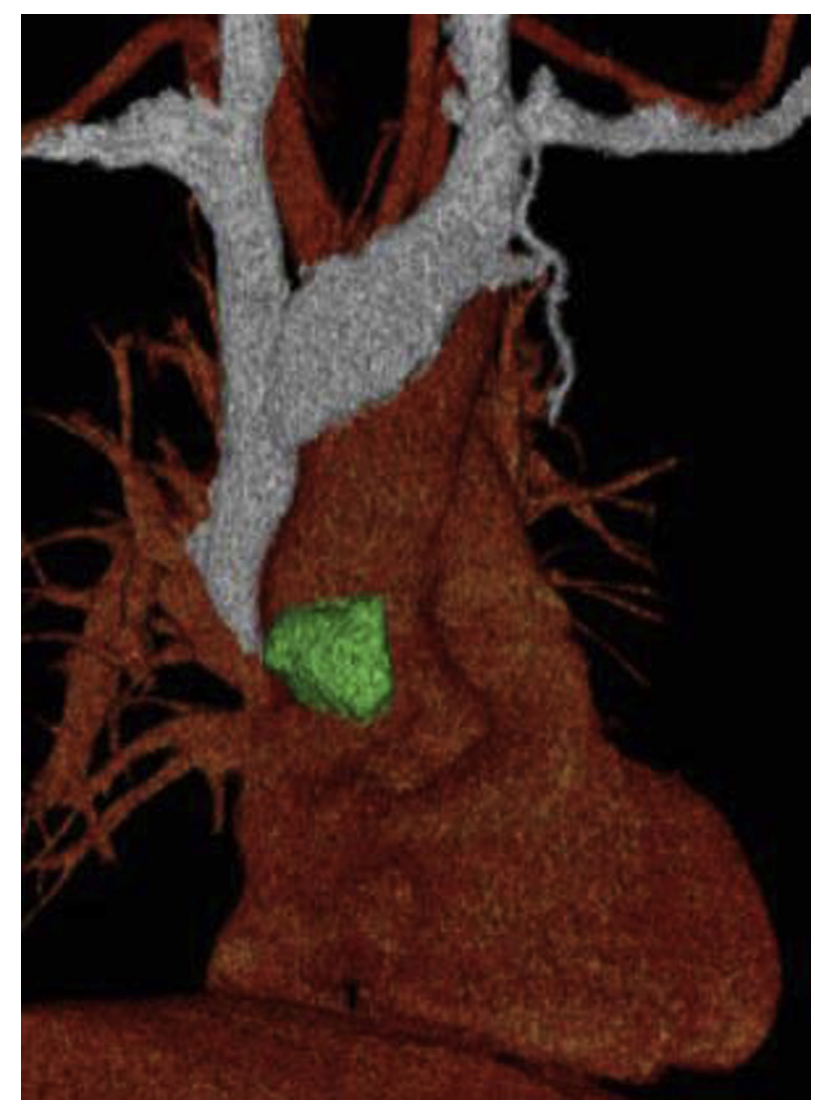

FIGURE 1. Reconstructed 3-dimensional CT image. The relationships between the nodule and the left brachiocephalic vein can be easily understood.

\section{DISCUSSION}

Surgical resection for thymoma is regarded as the principal treatment, and the completeness of resection is considered to be the most important determinant of the long-term survival of the patients with thymomas. ${ }^{1}$ Thymectomy is recommended for the treatment of thymoma. For thymectomy, median sternotomy is indispensable. Video-assisted thoracic surgery (VATS) has become widely used in thoracic surgery, such as in cases of mediastinal neoplasms or myasthenia gravis. ${ }^{2}$ Robotic surgery has also been used in surgery for anterior mediastinal tumors. ${ }^{3}$ Our institution has performed VATS thymectomy for the previous decade.

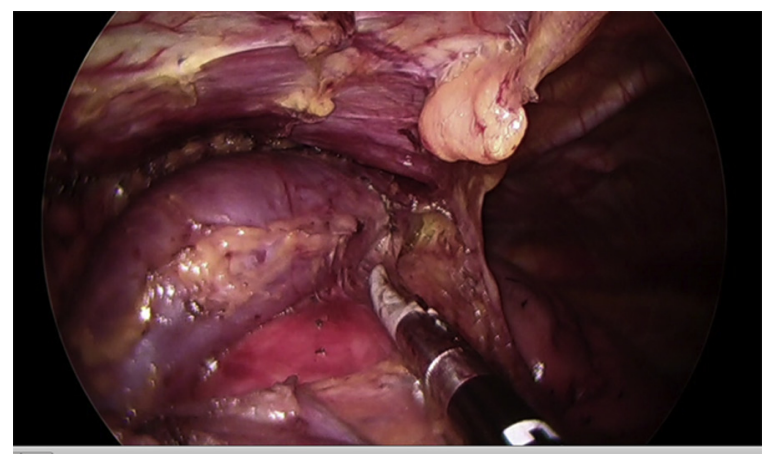

FIGURE 2. An intraoperative view of thymectomy.

We recently changed the approach of VATS thymectomy from the lateral approach to the subxiphoid approach because minimally invasive thymectomy could be achieved using this subxiphoid approach. Although the subxiphoid approach has been previously reported, ${ }^{4,5}$ through the invention of new instruments, thoracic surgeons can now perform minimally invasive thymectomy without any special techniques. The GelPOINT Mini and a lateral thoracic 5-mm port decrease the interference between the scope and instruments, which is the most challenging aspect of single-port surgery. An additional right lateral thoracic port also decreases the interference. The LigaSure Maryland can achieve single-hand exposure, sealing, and cutting of the vessels. Additionally, $\mathrm{CO}_{2}$ insufflation makes the procedures simpler by enlarging the working space. The improvement of the instruments and the surgical approach leads to minimally invasive thymectomy.

\section{References}

1. Masaoka A, Monden Y, Nakahara K, Tanioka T. Follow-up study of thymomas with special reference to their clinical stages. Cancer. 1981;48:2485-92.

2. Yim AP. Video-assisted thoracoscopic management of anterior mediastinal masses. Preliminary experience and results. Surg Endosc. 1995;9:1184-8.

3. Balduyck B, Hendriks JM, Lauwers P, Mercelis R, Ten Broecke P, Van Schil P Quality of life after anterior mediastinal mass resection: a prospective study comparing open with robotic-assisted thoracoscopic resection. Eur J Cardiothorac Surg. 2011;39:543-8.

4. Kido T, Hazama K, Inoue Y, Tanaka Y, Takao T. Resection of anterior mediastinal masses through an infrasternal approach. Ann Thorac Surg. 1999;67:263-5.

5. Suda T, Sugimura H, Tochii D, Kihara M, Hattori Y. Single-port thymectomy through an infrasternal approach. Ann Thorac Surg. 2012;93:334-6. 